\title{
Jet Lag From A Physics Point Of View
}

Wathiq Abdul-Razzaq, West Virginia University, USA

R. Dale Biller, Marshall University, USA

\begin{abstract}
While the common believe is that dark/light timing causes jet lag, we argue that physics, not the biological clock, may be the main reason for this air travel disorder. We explored the change in voltage in the human brain due to the added voltage induced by flying over the magnetic field of the Earth, and we think that this induced voltage in the brain is significant enough to cause neurological changes that may trigger jet lag or other illnesses.
\end{abstract}

Keywords: Jet Lag; Induced Voltage; Geomagnetism

\section{INTRODUCTION}

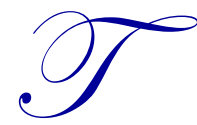

here are about 100 billion neurons in the brain with each neuron producing a voltage whose magnitude is 70 millivolts of electricity during operation (Barnett and Larkman, 2007). Each one of these neurons connect with about 1,000 other neurons or more in the human cortex to complete commands for a human to function in our everyday lives. The neurons in the human brain are surrounded by cells called Glia and are known as "nerve glue". There can be up to one trillion Glia cells in the human brain. The neurons are then connected by Synapses. These Synapses open and close in response to high or low voltage across the membrane or by a controlling chemical. These mechanisms are called voltage-gated ion-channels and chemically-gated ion-channels.

One aspect of human brain function depends on the level of voltage sustained in the human cortex (Sigworth, 2003). Thus we suspect that an induced voltage in the brain, due to the change of magnetic flux in the brain while flying through the magnetic field of the Earth, may affect the function of neurons and may cause or contribute to jet lag.

Jet lag is a physiological condition that can result from trans-meridian travel as on a jet plane. The condition is generally believed to be the result of disruption of the "light/dark" cycle that entrains the body's circadian rhythm (University of Washington, 2004). The symptoms vary depending on the person, but Jet Lag is known to cause anxiety, constipation, diarrhea, confusion, dehydration, headache, irritability, nausea, sweating, coordination problems, and even memory loss. The condition has also been reported to cause trouble with the heart and lack of resistance to illness (Cunha, Stöppler and Melissa, 2011).

The effect of jet lag was found to be strongest when crossing several meridian lines from east to west or west to east (E/W) as opposed to traveling on the same meridian line north to south or south to north (N/S). It is known that magnetic field (B) lines travel along the N/S direction. Therefore, traveling N/S along the magnetic field lines of the Earth is effectively traveling at a constant magnetic field, but traveling E/W is crossing separate areas of ever-changing magnetic field/intensity. We will see from equation (1) that when the magnetic field B is constant, there will be no induced voltage produced.

\section{CALCULATIONS}

The induced voltage $\boldsymbol{\varepsilon}$ generated in a loop due to changes in the magnetic flux $\Delta \phi$ in a time period $\Delta \mathrm{t}$ is given by Faraday's law (Serway and Vuille, 2012):

$|\boldsymbol{\varepsilon}|=\Delta \phi / \Delta \mathrm{t}$ 
with $\phi=\mathrm{BA} \cos \theta, \mathrm{B}$ is the magnetic field, $\mathrm{A}$ is the area of a loop, and $\theta$ is the angle between $\mathrm{B}$ and the normal to the plane of the loop.

Then equation (1) becomes:

$|\varepsilon|=\Delta(\mathrm{BA} \cos \theta) / \Delta \mathrm{t}$

If "A" represents an area of a loop of tissues in the brain (which is a constant value) and if $\theta$, defined above, is also constant as the direction of the trip (flight) does not change, then equation (2) becomes:

$|\varepsilon|=\mathrm{A} \cos \theta \Delta \mathrm{B} / \Delta \mathrm{t}$

It is clear from equation (3) that the induced voltage $\varepsilon$ in the brain can only be produced when there is a change in the magnetic field $\mathrm{B}$. Thus, traveling N/S along a magnetic field line does not produce induced voltage in the brain since $\mathrm{B}$ is constant along that line. However, traveling $\mathrm{E} / \mathrm{W}$ means crossing different magnetic field lines, and thus $\Delta \mathrm{B}$ will survive and an induced voltage $\varepsilon$ will be produced in the brain, the value of which will be calculated below:

The induced voltage $\boldsymbol{\varepsilon}$ can also be shown as (Serway and Vuille, 2012):

$|\varepsilon|=B L v \sin \theta$

where $\mathrm{L}$ is the length of a strip or rod travelling at a speed $\mathrm{v}$ and $\theta$ is the angle between $\mathrm{B}$ and $\mathrm{v}$.

For a strip of the brain of length 0.1 meter across the head of a person travelling in an airplane at the speed of $300 \mathrm{~m} / \mathrm{s}$ through the magnetic field of the Earth $\mathrm{B}=50 \mu \mathrm{T}, \varepsilon$ can be calculated using equation (4):

$|\boldsymbol{\varepsilon}|=\left(50 \times 10^{-6}\right)(0.1)(300) \sin \theta$

At the equator: $\theta=90$ degrees, thus

$|\varepsilon|=1.5 \times 10^{-3}$ Volts using equation (5)

At Arizona: $\theta=57$ degrees

$|\varepsilon|=1.258 \times 10^{-3}$ Volts using equation (5)

To understand the impact of this induced voltage on the brain, we compare it to the induced voltage produced by Transcranial Magnetic Stimulation (TMS) medical treatment in the following section.

\section{RESULTS}

Transcranial Magnetic Stimulation (TMS) and Repetitive Transcranial Magnetic Stimulation (rTMS) are noninvasive techniques used in hospitals to stimulate regions of the human brain (Barker, Jalinous, and Freeston, 1985). They are recent medical procedures for mental illness that use pulses of magnetic field (changing magnetic field) to induce voltage in the human brain and have proven to be effective in treatment of depression. During the TMS treatment, the magnetic field $\mathrm{B}$ in the cortex is varied from 0 to $0.5 \mathrm{~T}$ in about $100 \mathrm{~ms}$. The induced voltage $\varepsilon$ within a circle of brain tissue of radius $1.6 \mathrm{~mm}$ of the area responsible for depression can be calculated using equation (1):

$|\boldsymbol{\varepsilon}|=\Delta \phi / \Delta \mathrm{t}=(\Delta \mathrm{B} \mathrm{A}) / \Delta \mathrm{t}=(0.5-0) \pi\left(1.6 \times 10^{-3}\right)^{2} /\left(100 \times 10^{-3}\right)=0.4 \times 10^{-3}$ Volts 
This $0.4 \mathrm{mV}$ resulting induced voltage in the brain is sufficient to stimulate neuronal activity and cure depression (Serway and Vuille, 2012). Our predicted induced voltage of $1.5 \mathrm{mV}$ or $1.25 \mathrm{mV}$ calculated above, while flying, is about four times the value produced by TMS. Thus, we strongly believe that the induce voltage in the brain produced while flying should be considered as a factor in understanding jet lag or other illnesses, such as the weakening of the immune system while flying.

Strafella, Paus, Barrett, and Dagher (2001) performed an MRI scan on the human brain and found Dopamine release in the prefrontal cotes of the brain during and after a 30-minute rTMS study. The prefrontal cortex is responsible for movement, learning, and motivation in the prefrontal cortex. The release of Dopamine in the prefrontal cortex can be associated with illnesses like Parkinson's disease, schizophrenia, and drug addiction (Strafella, Paus, Barrett, and Dagher, 2001). While patient's need three treatments to express a change in brain function of a total time maximum 30 minutes, flying in a jet plane can last for nine hours, or more for international flights hours.

\section{MAGNETIC FIELD MEASUREMENTS INSIDE AN AIRPLANE}

The purpose of this experiment was to see if the skin of an aircraft has any shielding effect on the magnetic field of the Earth. We used the G816 portable proton magnetometer manufactured by Geometrics that has a nanoTesla range to measure the magnetic field of the Earth while flying inside a Cessna 172 aircraft. We flew from Morgantown Municipal Airport located in the state of West Virginia, USA, at an altitude of up to 3,500 ft. The magnetic field measurements inside the aircraft showed no net decrease in the Earth's magnetic field readings before and after entering the plane and while flying. If anything, we learned that upon starting, the airplane actually increased the magnetic field inside the cockpit as this particular type of plane has a magneto for engine charging that produces an additional magnetic field at starting - jet planes do not have a magneto. Thus, our first point of measurement at take off of the engine was higher than any other measurement due to the magneto. The magnetic field measurements from inside the aircraft are shown in Figure 1. It is clear from the figure that the shielding of the aircraft, which is similar to the shielding of a jet plane, has no significant effect on the magnetic field of the Earth. This means that people inside the aircraft experience the typical value of the magnetic field of the Earth of about 50 $\mu \mathrm{T}$.

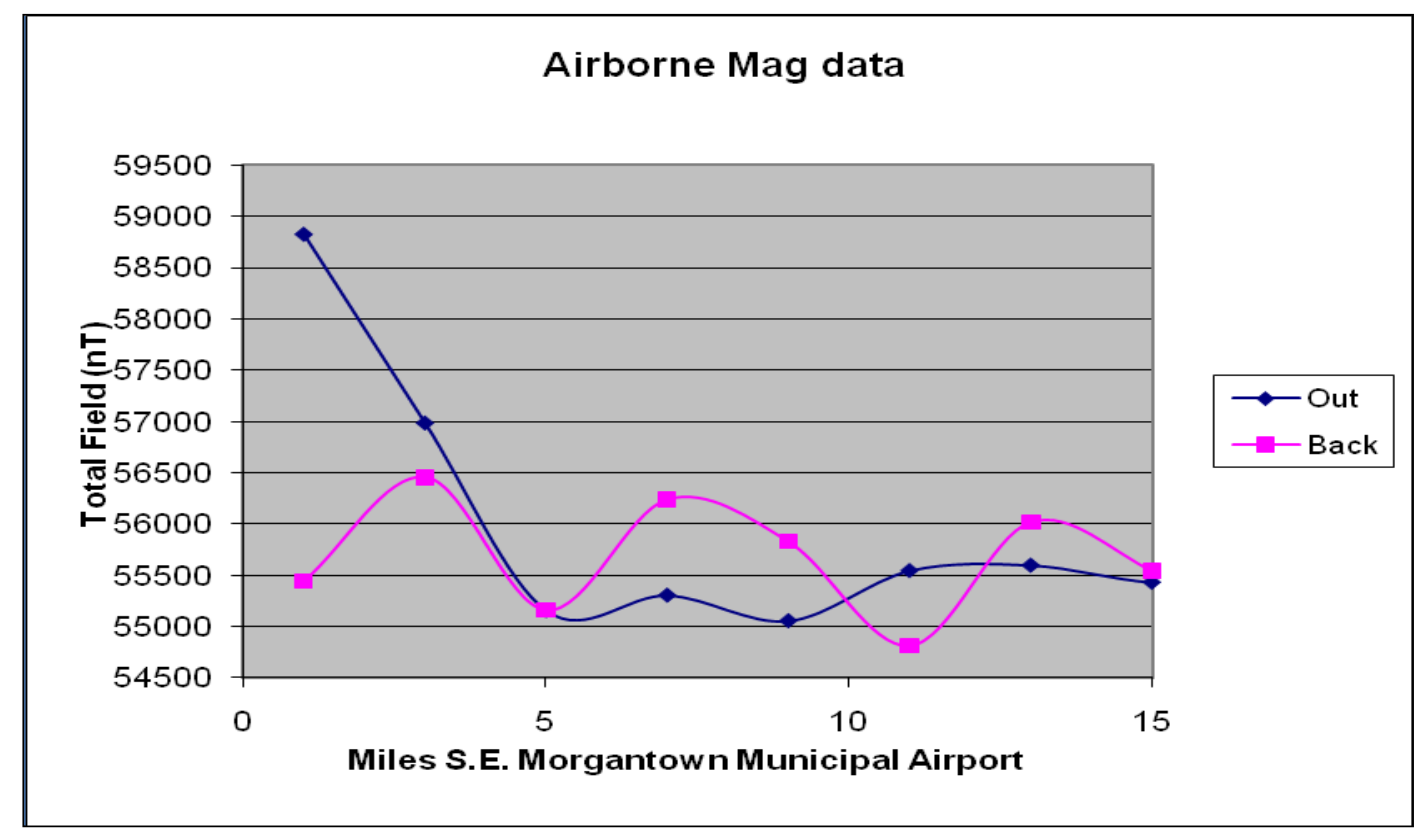

Figure 1: The Airborne Magnetic Field in NanoTeslas (nT) While In-flight Aboard A Cessna 172 


\section{CONCLUSION}

As a person travels at high speed while flying, an appreciative induced voltage can be produced on the human brain due to the change in the magnetic flux brought on by the magnetic field of the Earth and motion. We have estimated that induced voltage and found it to be larger than the induced voltage produced in the brain by the medical treatment Transcranial Magnetic Stimulation (TMS). This treatment targets a particular part of the brain; for example, the part responsible for depression, and can have an effect on the brain even for a 30-minute treatment. While the induced voltage produced during flights affect the whole brain and can last for over 9 hours during international flights. We are raising the question; if the induced voltage in the brain that results from prolonged exposure to the Earth's magnetic field at a high speed can alter the function of the human brain and cause jet lag, or even other illnesses, what other explanation is needed? What strengthens our believe is our suggestion that jet lag is strongest when crossing several meridian lines east/west as opposed to traveling on the same meridian line north/south. This suggests dissimilar induced voltages for the two cases as the magnetic field lines of the Earth follow the south/north direction. We suggest a clinical study to understand jet lag that focuses on induced voltage as we are not aware of such a study in the literatures.

\section{AUTHOR INFORMATION}

Dr. Wathiq Abdul-Razzaq received his Ph.D. in 1986 from the University of Illinois at Chicago. He completed his post-doctorate work at Michigan State University. Currently he is Professor of Physics and Director of Introductory Physics Curriculum at West Virginia University. He has had research activities in diverse areas including studies of magnetic and transport properties of thin films and multilayers, studies of magnetic nanoparticles, applied studies related to health and environment, and research in education. Full address: Physics Department, West Virginia University, 135 Willey Street, PO Box 6315, Morgantown, WV 26506, Tel: 304-293-5045. E-mail: wabdulra@wvu.edu (Corresponding author)

R. Dale Biller received his Bachelor of Science degree in Safety Engineering and his Master of Science degree in Physical Science/ Geology from Marshall University, West Virginia. He is currently working for EQT Energy as a Geophysical Analyst. He also does research in collaboration with Dr. W.Abdul-Razzaq that involves geology, physics and education. E-mail: dalebiller@yahoo.com

\section{REFERENCES}

1. $\quad$ Barnett M.W., Larkman P.M. (2007) The action potential. Pract Neurol 7 (3), 192-197.

2. Sigworth, F.J. (2003). Structural biology: life's transistors. Nature 423:21-22.

3. University Of Washington (2004, May 18). Artificial Light-dark Cycles Expose Circadian http://www.eurekalert.org/pub_releases/2004-05/uow-alc051104.php

4. Cunha, John P.; Stöppler, \& Melissa Conrad (2011) http://www.medicinenet.com/jet_lag/article.htm

5. Serway R., Vuille C. (2012) P 692, 697 College Physics, $9^{\text {th }}$ Edition : BROOKS/COLE ISBN 978-0-84006206-2

6. $\quad$ Barker A.T., Jalinous R., Freeston I.L. (1985) "Non-invasive magnetic stimulation of human motor cortex." Lancet 1: 1106-1107.

7. $\quad$ Serway R, Vuille C; (2012) Pg. 716 College Physics, $9^{\text {th }}$ Edition : BROOKS/COLE ISBN 978-0-84006206-2

8. Strafella A., Paus T., Barrett J., Dagher A. (2001) Repetitive transcranial magnetic stimulation of the human prefrontal cortex induces dopamine release in the caudate nucleus, The Journal of Neuroscience, 21:RC157, 1-4. 